\title{
CONHECIMENTO SOBRE SAÚDE SEXUAL E REPRODUTIVA EM ESTUDANTES DO ENSINO SUPERIOR
}

\author{
Maria Jose Santos \\ Universidade de Trás-os-Montes e Alto Douro, Escola Superior de Saúde, Vila Real, Portugal UICISA: E \\ Research Centre - EsenfC/ESSIPV \\ mjsantos@utad.pt \\ Paula Nelas \\ Instituto Politécnico de Viseu, Escola Superior de Saúde de Viseu, Viseu, Portugal UICISA: E Research Centre - \\ EsenfC/ESSIPV \\ pnelas@gmail.com \\ Manuela Ferreira \\ Instituto Politécnico de Viseu, Escola Superior de Saúde de Viseu, Viseu, Portugal UICISA: E Research Centre - \\ EsenfC/ESSIPV \\ mmcferreira@gmail.com
}

Recepción Artículo: 27 octubre 2021

Admisión Evaluación: 27 octubre 2021

Informe Evaluador 1: 28 octubre 2021

Informe Evaluador 2: 29 octubre 2021

Aprobación Publicación: 30 octubre 2021

\section{RESUMO}

Introdução: A promoção da saúde sexual e reprodutiva (SSR) dos estudantes do Ensino Superior continua a ser uma prioridade das políticas de saúde pública, dado que vários estudos têm documentado prevalências elevadas de comportamentos de risco sexual neste grupo. 0 conhecimento é um conceito central das teorias comportamentais, considerado como um pré-requisito fundamental para uma educação sexual de sucesso. Objetivos: Caracterizar o conhecimento sobre SSR dos estudantes do Ensino Superior e analisar a sua associação com comportamentos promotores da SSR. Metodologia: Foi realizado um estudo transversal analítico, numa amostra de 1946 estudantes, de uma Universidade do norte de Portugal, com idade média de 21 anos (20,74 $\pm 2,32$ ). Na recolha de dados foi utilizado um questionário de caracterização sociodemográfica e dos comportamentos de SSR e o Inventário de Conhecimento Sobre SSR, organizado em quatro áreas temáticas (fisiologia da reprodução, contraceção, infeções sexualmente transmissíveis (IST), vigilância da saúde). Os dados foram analisados com recurso à estatística descritiva e inferencial (ANOVA). Resultados: 0 nível de conhecimento sobre SSR é mediano (22,27 $\pm 5,79)$, a área de maior conhecimento é a contraceção e a de menor conhecimento as IST e vigilância de saúde. 0 nivel de conhecimento é mais elevado no sexo feminino $(p<0,05)$, estudantes mais jovens $(\mathrm{p}=0,005)$ e que frequentam cursos das áreas das ciências da vida e saúde $(\mathrm{p}<0,001) .0$ conhecimento está associado positivamente com uma maior adesão a comportamentos de contraceção $(p=0,03)$, utilização dos ser- 


\section{CONHECIMENTO SOBRE SAÚDE SEXUAL E REPRODUTIVA EM ESTUDANTES DO ENSINO SUPERIOR}

viços de SSR $(p<0,01)$ e menor adesão a comportamentos de risco sexual, nomeadamente 0 consumo de álcool $(p<0,001)$, drogas $(p<0,001)$ e parceiros ocasionais $(p<0,001)$. Conclusões: Aumentar 0 conhecimento e dos estudantes do Ensino Superior sobre SSR parece ser importante, para melhorar comportamentos de proteção e diminuir o risco sexual e reprodutivo. As universidades podem ser com contexto privilegiado para intervenção em educação sexual e reprodutiva.

Palabras chave: conhecimento; estudantes; ensino superior; saúde sexual e reprodutiva

\section{ABSTRACT}

Knowledge about sexual and reproductive health in higher education students. Introduction: The promotion of sexual and reproductive health (SRH) of higher education students continues to be a priority of public health policies, as several studies have documented high prevalence of sexual risk behavior in this group. Knowledge is a central concept of behavioral theories, considered as a fundamental prerequisite for successful sex education. Objectives:. Characterize the knowledge about SRH of students in Higher Education and analyze its association with behaviors that promote SRH. Methodology: An analytical cross-sectional study was carried out in a sample of 1946 students from a University in the north of Portugal, with a mean age of 21 years (20.74 \pm 2.32 ). A questionnaire of sociodemographic characterization and SSR behaviors and the SRH Knowledge Inventory were used to collect data, organized into four thematic areas (physiology of reproduction, contraception, sexually transmitted infections (STI), health surveillance). Data were analyzed using descriptive and inferential statistics (ANOVA). Results: The level of knowledge about SSR is medium (22.27 \pm 5.79$)$, the area of greatest knowledge is contraception and the least knowledge of STIs and health surveillance. The level of knowledge is higher in females $(p<0.05)$, younger students $(p=0.005)$ and who attend courses in the areas of life sciences and health $(p<0.001)$. Knowledge is positively associated with greater adherence to contraceptive behaviors $(p=0.03)$, use of SRH services $(p<0.01)$ and lower adherence to sexual risk behaviors, including alcohol consumption $(p<0.001)$, drugs $(p<0.001)$ and occasional partners $(p<0.001)$. Conclusions: Increasing knowledge and knowledge of higher education students about SRH seems to be important to improve protective behaviors and decrease sexual and reproductive risk. Universities can be a privileged context for intervention in sexual and reproductive education.

Keywords: knowledge; college; students; sexual and reproductive health

\section{INTRODUÇÃO}

A saúde sexual e reprodutiva (SSR), é uma parte essencial da saúde pública e pode influenciar consideravelmente 0 bem-estar geral e a qualidade de vida dos indivíduos. Não obstante, a melhoria significativa dos indicadores relativos à SSR nas ultimas décadas, os estudantes do Ensino Superior são habitualmente esquecidos nos diagnósticos e intervenções de saúde pública, em virtude do entendimento de que este grupo, comparativamente com os adolescentes, apresenta maior conhecimento e maior capacidade para tomar decisões relacionadas com a sexualidade (Matos, Reis, Ramiro \& Equipa Aventura Social, 2011). Assim a promoção da SSR dos estudantes do ensino superior deve constituir uma prioridade das políticas de saúde pública, dado que vários estudos têm documentado prevalências elevadas de comportamentos de risco sexual neste grupo (Hartney et al., 2015). Estes riscos estão associados a práticas de sexo inseguro relacionados com uma utilização inconsistente do preservativo (Asare, 2015; Santos, Ferreira, Duarte \& Ferreira et al., 2017), à utilização de álcool e drogas associadas às relações sexuais e parceiros ocasionais (Kuperberg \& Padgett, 2015; Khadr et al., Oliveira et al, 2017; Kilwein \& Looby, 2018; Garcia et al., 2019), comportamentos considerados quase normativos sobretudo no contexto da noite e das festas académicas.

Se em Portugal os dados são escassos e pouco sistematizados, alguns dados dos relatórios de vigilância epidemiológica sobre os comportamentos de risco para a saúde a nível internacional, apontam para a manutenção de elevadas taxas de prevalência das IST entre os jovens (Forsyth et al., 2018; Kann et al., 2019;). Apesar da infeção por VIH/SIDA ser a mais severa, muitas outras IST, tais como a Chlamydia, o Vírus Papiloma Humano (HPV), 
a sífilis e a gonorreia podem estar associadas a inúmeras complicações reprodutivas, a curto e a longo prazo e, em particular, na fertilidade (Shepherd et al., 2010). A contraceção e a prevenção de IST constituem duas importantes vertentes da saúde reprodutiva e a sua interação é inequívoca, pelo que a realização da sua abordagem conjunta junto dos jovens, representa mais uma oportunidade para educar e prevenir riscos para a SSR. A prática de sexo seguro implica não só o conhecimento dos métodos contracetivos, mas também das estratégias de redução da incidência das IST (Pama \& Neves, 2013).

Compreender os fatores que influenciam os comportamentos SSR dos jovens do ensino superior é relevante, uma vez que estes podem ter um impacto significativo na tomada de decisão e os programas educacionais podem diminuir significativamente os riscos sexuais associados a esses fatores (Kirby et al., 2011). De acordo com Picot et al. (2012), nos jovens adultos o risco sexual tem sido associado à falta de consciência, conhecimento insuficiente sobre comportamentos protetores e normas sociais.

0 conhecimento fornece uma base para a ação humana e é um conceito central em muitas teorias comportamentais, usado como base teórica na construção de programas de educação sexual. Embora seja consensual que 0 conhecimento por si só não determine o comportamento, ele é considerado um pré-requisito importante porque fornece a fundamentação para muitos valores, atitudes, perceções de normas e competências que, por sua vez, permitem a concretização do comportamento sexual (Kirby et al., 2011; Kabwama \& Berg-Beckhoff, 2015).

Neste sentido, alguns autores afirmam que as intervenções na área da educação sexual que melhoram os conhecimentos e a consciência sobre os riscos sexuais, podem influenciar positivamente as atitudes e os comportamentos face às medidas de prevenção (Gerend \& Shepherd, 201; Kritsotakis et al., 2016; Bruni et al., 2015;). Vários estudos sugerem que um maior conhecimento e atitudes mais positivas estão correlacionados com uma utilização mais consistente do preservativo e que os estudantes mais informados tendem a não aceitar o risco sexual (Asare, 2015; Reis et al., 2013; Sales et al, 2016). 0 acesso a informação de qualidade pode contribuir para um aumento da prática de sexo seguro e uma utilização mais responsável dos métodos contracetivos (Sales et al, 2016; Santos et al., 2018; Castro et al., 2020).

\section{OBJETIVOS DA INVESTIGACÃO}

Caracterizar o conhecimento sobre SSR dos estudantes do Ensino Superior e analisar a sua associação com comportamentos promotores da SSR.

\section{AMOSTRA E/OU PARTICIPANTES}

Foi realizado um estudo transversal, descritivo-correlacional, numa amostra não probabilística de 1946 estudantes, com predomínio do género feminino (64\% raparigas e 36\% rapazes), com média de idades de 21 anos $(20,74 \pm 2,32)$, que frequentavam diversas áreas científicas de uma universidade do norte de Portugal. A maioria dos estudantes era de nacionalidade portuguesa $(97,3 \%)$, solteiros $(97,6 \%)$, proveniente da cidade $(40 \%)$, de famílias com baixo rendimento ( $57 \%, \leq 2$ salários mínimos), baixo nível de escolaridade (54,5\% das mães e $61,6 \%$ dos pais têm apenas o ensino básico) e com profissões pouco diferenciadas.

Os participantes são provenientes de curso de uma das 5 escolas da Universidade (4 de natureza universitária e uma de natureza politécnica) e na sua maioria encontravam-se a frequentar $02^{\circ}$ ou $3^{0}$ ano de uma licenciatura. Aproximadamente dois terços $(63,4 \%)$ eram estudantes de cursos de ciências da vida.

\section{METODOLOGIA E/OU INSTRUMENTOS UTILIZADOS}

Na recolha de dados foi utilizado um questionário de autopreenchimento, que foi aplicado em sala de aula, e permitiu recolher informação sobre:

- Características sociodemográficas: sexo, idade, nacionalidade, local onde cresceu, área científica de estudos, nível de escolaridade dos pais ( 9 anos, 10 a 12 anos, ensino superior), rendimento familiar mensal (menos 2 salários mínimos; entre 2 e 4 salários mínimos, mais 4 salários mínimos) 


\section{CONHECIMENTO SOBRE SAÚDE SEXUAL E REPRODUTIVA EM ESTUDANTES DO ENSINO SUPERIOR}

- Comportamentos sexuais e reprodutivos: relações sexuais nos últimos 12 meses, tipo de relação, uso de um método contracetivo, tipo de método utilizado, fontes de informação sobre sexualidade e contraceção, comportamentos de risco (sexo com álcool, drogas, parceiros ocasionais, utilização consistente do preservativo).

- Nível de conhecimento em SSR: foi avaliado por meio de um inventário de autorrelato composto por 44 questões, construído especificamente apara o estudo, com base no conhecimento dos ambientes sociais dos alunos e em uma revisão da literatura sobre SSR. 0 instrumento permitiu a organização da informação em quatro dimensões: (1) fisiologia da reprodução (2) métodos de contraceção, (3) IST e medidas de prevenção do risco sexual e (4) recomendações sobre vigilância de SSR. Os itens foram avaliados usando três opções de resposta: verdadeiro, falso e não sei. As respostas corretas foram codificadas como um, e as respostas incorretas ou incertas foram codificadas como zero. A pontuação total variou de 0 a 44 pontos. A confiabilidade do inventário de conhecimento foi avaliada usando o coeficiente da fórmula 20 de Kuder-Richardson (KR20), um análogo ao alfa de Cronbach e é usado como uma medida de consistência interna em estudos com escalas dicotômicas (Hair, Black, Babin \& Anderson, 2014). 0 resultado foi KR20 $=0,78$, confirmando a adequada consistência interna do instrumento.

A participação no estudo foi de carácter voluntário e foram asseguradas as recomendações éticas, nomeadamente a confidencialidade dos resultados e 0 anonimato dos participantes. 0 estudo foi autorizado pela Comissão de Ética da Universidade onde foi realizado e salvaguardou os princípios ético-deontológicos consignados na Declaração de Helsínquia, nos diferentes momentos do processo de investigação.

0 tratamento estatístico dos dados foi efetuado com o programa Statistical Package for the Social Sciences (SPSS, Inc, Chicago, IL, USA), na versão 24.0. Na análise de dados foi utilizada a estatística descritiva: frequência ou medidas de tendência central e de dispersão, conforme a natureza dos dados. A associação entre as características sociodemográficas, académicas, comportamentos sexuais e o conhecimento sobre SSR foram analisadas usando análise de variância simples (ANOVA). A localização de diferenças entre médias foi feita com 0 teste Tukey. Em todas as estatísticas inferenciais, foi utilizado como limite de significância 0 valor de $p<0,05$.

\section{RESULTADOS}

No que respeita aos comportamentos sexuais e reprodutivos, a maioria dos estudantes $(76,9 \%)$ refere ter tido relações sexuais coitais nos últimos 12 meses, mais de metade (68\%) das quais aconteceram no âmbito de uma relação amorosa. A grande maioria dos estudantes $(96,7 \%)$, refere ter usado contraceção sendo o método mais utilizado o hormonal (43,7\%) e o preservativo (21,8\%), contudo apenas 39,5\% dos participantes mencionaram usar o preservativo de forma consistente.

Uma percentagem significativa de estudantes reporta também o envolvimento em comportamentos de risco sexual nos últimos 12 meses, nomeadamente relações sexuais associadas ao consumo de álcool (33,0\%) ou drogas $(9,7 \%)$ e múltiplos parceiros ocasionais $(32,0 \%)$.

A grande maioria $(77,8 \%)$ dos estudantes nunca utilizou os serviços de SSR, mas as raparigas utilizavam mais estes serviços que os rapazes ( $=30,4 \%$ vs $=7,6 \%, p<0,0001$ ). As dificuldades no acesso estavam sobretudo relacionadas com os horários de funcionamento, localização dos serviços, demora na marcação das consultas e as questões de anonimato e confidencialidade. No que respeita à vigilância de saúde, a maioria (85,5\%) nunca realizou 0 teste do $\mathrm{VIH}$ e apenas $66,9 \%$ das raparigas foram vacinadas para 0 vírus do papiloma humano (HPV), sendo que 6,4\% desconhece mesmo essa necessidade. Uma grande maioria das raparigas (65,1\%) nunca realizou citologia vaginal e das que realizam, 63,7\% fazem o teste anualmente. Mais de metade das raparigas $(56,6 \%)$ não faz autoexame da mama e cerca de metade dos rapazes $(47,9 \%)$ não realiza 0 e 0 autoexame do testículo, e 32,2\% desconhece a sua importância.

As fontes mais comuns de informação sobre SSR foram amigos (46,1\%), internet $(45,3 \%)$ e professores (42,7\%), contatando-se que os profissionais de enfermagem têm papel secundário na transmissão das informações $(24,2 \%)$. 
0 conhecimento dos estudantes do ensino superior sobre SSR no global é mediano $(22,76 \pm 5,79$, pontos num score total de 44), e é na dimensão conhecimento sobre contraceção que os estudantes apresentam níveis mais elevados (8,42 $\pm 2,44$, pontuação máxima de 16) e na dimensão conhecimento sobre ISTs/risco sexual $(6,05 \pm 2,73)$ e vigilância de saúde $(1,43 \pm 0,95)$ que revelam pontuações mais baixos (Tabela 1.). Sobretudo na última dimensão composta por quatro questões relacionadas com vacinação contra o HPV e citologia vaginal.

Table 1.

Análise da influência dos fatores sociodemográficos e acadêmicos no nível de conhecimento em saúde sexual e reprodutiva.

\begin{tabular}{|c|c|c|c|c|c|}
\hline \multirow[b]{2}{*}{ Variáveis } & \multicolumn{5}{|c|}{ Conhecimento sobre SSR } \\
\hline & $\begin{array}{c}\text { Fisiologia da } \\
\text { Reprodução } \\
\qquad(0-8)^{\Psi}\end{array}$ & $(0-16)^{\Psi}$ & $(0-16)^{\Psi}$ & $\begin{array}{c}\begin{array}{c}\text { Vigilância } \\
\text { de Saúde }\end{array} \\
(0-4)^{\Psi}\end{array}$ & $(0-44)^{\Psi}$ \\
\hline $\begin{array}{l}\text { Total } \\
\text { estudantes }\end{array}$ & $3,86 \pm 1,67$ & $8,42 \pm 2,44$ & $6,05 \pm 2,73$ & $1,43 \pm 0,95$ & $22,76 \pm 5,79$ \\
\hline \multicolumn{6}{|l|}{ Sexo } \\
\hline Male $(n=690)$ & $3,21 \pm 1,65$ & $7,37 \pm 2,62$ & $8,50 \pm 2,95$ & $1,03 \pm 0,90$ & $20,11 \pm 6,07$ \\
\hline \multirow[t]{2}{*}{$\begin{array}{l}\text { Female } \\
(n=1246)\end{array}$} & $4,22 \pm 1.56$ & $9,01 \pm 2,12$ & $9,36 \pm 2,55$ & $1,65 \pm 0,91$ & $24,24 \pm 5,05$ \\
\hline & $\mathrm{p}<0.001$ & $\mathrm{p}<0,001$ & $\mathrm{p}<0,001$ & $\mathrm{p}<0,001$ & $\mathrm{p}<0,001$ \\
\hline \multicolumn{6}{|l|}{ Idade } \\
\hline$\leq 19(\mathrm{n}=619)$ & $3,94 \pm 1,59 b$ & $8,63 \pm 2,39 b$ & $9,10 \pm 2,72$ & $1,50 \pm 0,94$ & $23,17 \pm 5,60 b$ \\
\hline $20-24(n=1187)$ & $3,86 \pm 1,69 b$ & $8,40 \pm 2,43 b$ & $9,05 \pm 2,70$ & $1,41 \pm 0,95$ & $22,72 \pm 5,83 b$ \\
\hline \multirow[t]{2}{*}{$\geq 25(\mathrm{n}=139)$} & $3,53 \pm 1,70 \mathrm{a}$ & $7,69 \pm 2,59 \mathrm{a}$ & $8,85 \pm 3,05$ & $1,32 \pm 0,95$ & $21,39 \pm 6,08 \mathrm{a}$ \\
\hline & $\mathrm{p}=0,038$ & $\mathrm{p}<0,001$ & $\mathrm{p}=0,624$ & $\mathrm{p}=0,061$ & $\mathrm{p}=0,005$ \\
\hline \multicolumn{6}{|l|}{ Àrea de estudos } \\
\hline Ciências e Saúde ( $\mathrm{n}=1233$ ) & $4,10 \pm 1,63$ & $8,80 \pm 2,30$ & $9,60 \pm 2,52$ & $1,52 \pm 0,96$ & $24,02 \pm 5,33$ \\
\hline \multirow[t]{2}{*}{ Outras $(n=713)$} & $3,44 \pm 1,65$ & $7,75 \pm 2,53$ & $8,10 \pm 2,82$ & $1,27 \pm 0,92$ & $20,57 \pm 5,89$ \\
\hline & $\mathrm{p}<0,001$ & $\mathrm{p}<0,001$ & $\mathrm{p}<0,001$ & $\mathrm{p}<0,001$ & $\mathrm{p}<0,001$ \\
\hline
\end{tabular}

SSR - Saúde Sexual e Reprodutiva; ${ }^{\Psi}$ - Scores mínimos e máximos do instrumento; IST's - Infeções de Transmissão Sexual; abc - As médias na mesma coluna para a variável idade seguidas de letras diferentes são diferentes $(\mathrm{p}<0.05)$.

As raparigas apresentaram níveis médios de conhecimento em SSR mais elevados $(p<0,001)$ que os rapazes, diferenças significativas que também foram observadas no conhecimento entre estudantes de diferentes faixas etárias. Os estudantes mais jovens apresentaram níveis mais elevados de conhecimento $(p<0,05)$ para os 
aspetos gerais da fisiologia da reprodução, contraceção e, consequentemente, apresentaram níveis de conhecimento global mais elevados. A área científica de estudo também influenciou o conhecimento, observando-se que os estudantes dos cursos das áreas das ciências da vida e da saúde apresentaram níveis de conhecimento mais elevados $(p<0,001)$ do que aqueles que estudavam outras áreas.

Analisando a relação entre os comportamentos sexuais e o conhecimento em SSR (Tabela 2.), observou-se que a utilização de contraceção, a realização do teste HIV e a utilização dos serviços de SSR estavam associados de forma significativa com o conhecimento, assim como os três comportamentos de risco sexual $(p<0,001)$. Os estudantes com níveis mais baixos de conhecimento referem maior envolvimento nos três comportamentos de risco sexual estudados. A realização do teste HIV também se mostrou dependente do conhecimento $(p=0,031)$.

Tabela 2.

Análise da relação entre os comportamentos de SSR e o conhecimento em SSR

\begin{tabular}{|c|c|c|}
\hline & \multicolumn{2}{|l|}{ Conhecimento de SSR } \\
\hline & Média \pm DP & $\mathbf{p}$ \\
\hline \multicolumn{3}{|l|}{ Vida sexual ativa } \\
\hline $\operatorname{Sim}(n=1496)$ & $22,77 \pm 5,78$ & 0,930 \\
\hline Não (n=449) & $22,74 \pm 5,86$ & \\
\hline \multicolumn{3}{|l|}{ Usa contraceção } \\
\hline $\operatorname{Sim}(n=1447)$ & $22,86 \pm 5,76$ & 0,003 \\
\hline Não $(\mathrm{n}=56)$ & $20,44 \pm 6,02$ & \\
\hline \multicolumn{3}{|c|}{$\begin{array}{l}\text { Utilização consistente do } \\
\text { preservativo }\end{array}$} \\
\hline $\operatorname{Sim}(n=591)$ & $22,69 \pm 5,81$ & 0,625 \\
\hline Não (n=906) & $22,84 \pm 5,76$ & \\
\hline \multicolumn{3}{|l|}{ Sexo com álcool } \\
\hline $\operatorname{Sim}(n=511)$ & $22,00 \pm 5,82$ & 0,001 \\
\hline Não (n=1011) & $23,06 \pm 5,77$ & \\
\hline \multicolumn{3}{|l|}{ Sexo com drogas } \\
\hline $\operatorname{Sim}(n=147)$ & $20,41 \pm 5,98$ & $<0,001$ \\
\hline Não (n=1361) & $22,96 \pm 5,74$ & \\
\hline \multicolumn{3}{|c|}{ Sexo com parceiros ocasionais } \\
\hline $\operatorname{Sim}(n=482)$ & $21,81 \pm 5,68$ & $<0,001$ \\
\hline Não (n=1023) & $23,14 \pm 5,78$ & \\
\hline \multicolumn{3}{|c|}{ Utiliza serviços de SSR } \\
\hline $\operatorname{Sim}(n=432)$ & $24,78 \pm 4,95$ & $<0,001$ \\
\hline Não (n=1449) & $22,18 \pm 5,88$ & \\
\hline \multicolumn{3}{|l|}{ Fez teste VIH } \\
\hline $\operatorname{Sim}(n=283)$ & $23,37 \pm 5,89$ & $<0,031$ \\
\hline Não (n=1663) & $22,66 \pm 5,76$ & \\
\hline
\end{tabular}

SRR - Saúde Sexual e Reprodutiva; DP- Desvio padrão.

\section{DISCUSSÃO}

Os resultados obtidos permitem afirmar que maioria dos estudantes são sexualmente ativos, mais de metade dos participantes tem um relacionamento amoroso atual e mantém relações sexuais com o companheiro, revelando na sua maioria preocupação com utilização de um método contracetivo nas relações sexuais. A tendência das raparigas utilizarem maioritariamente contraceção hormonal, prende-se com a sua elevada eficácia e facilidade de utilização, uma vez que são elas que, na maior parte das situações, assumem a responsabilidade pela 
escolha do método contracetivo. Por sua vez, o método contracetivo mais utilizado pelos rapazes é o preservativo, muito recomendado nesta faixa etária, dado carácter espontâneo e muitas vezes não planeado das relações sexuais (Tyler et al., 2014). Os estudantes mais velhos são os que apresentam um comportamento contracetivo mais inconsistente, sobretudo quando envolvidos numa relação amorosa de maior duração. 0 abandono do uso do preservativo pode resultar da alegada confiança no parceiro sexual, crenças na redução no prazer (Santos et al., 2017). Outros autores referem ainda a procura de novas sensações sexuais, mais marcada em rapazes mais velhos (Voisin, King, Schneider, Diclemente \& Tan, 2012) e a baixa perceção de risco de transmissão de IST (Asare, 2015; Castro et al., 2020).

Para além de se assegurar a disponibilidade dos contracetivos, é essencial reforçar a educação contracetiva dos jovens no sentido de potenciar a sua capacidade de escolha, investindo também na formação específica dos profissionais de saúde, uma vez que diversos estudos comprovam que um bom aconselhamento contracetivo melhora a adesão e diminui a taxa de descontinuação (Gómez-Olivé, Kahn, Tollman \& Norris, 2014).

Os estudantes universitários têm níveis moderados de conhecimento em relação à SSR, conforme relatado de forma consistente na literatura (Reis et al., 2013; Sohbet \& Geçici, 2014), contudo as raparigas que apresentam níveis elevados de conhecimento. Esta tendência relacionada com o género pode ser justificada pelo maior investimento dos serviços de saúde nas necessidades das mulheres, pois, devido aos múltiplos determinantes biológicos e sociais, elas são consideradas como mais vulneráveis para os diversos riscos sexuais e reprodutivos (Pama \&Neves, 2013). 0 maior nível de conhecimento sobre SSR das raparigas também pode estar relacionado com as fontes de informação que utilizam (profissionais de saúde e professores), mais formais que as utilizadas pelo rapazes (amigos e internet). São também as raparigas que utilizavam mais os serviços de SSR, revelando que as raparigas comparativamente como s rapazes demonstram quase sempre uma maior preocupação com as questões de saúde (Santos et al., 2018).

0 baixo nível de conhecimento em SSR está associado negativamente uma menor adesão a comportamentos de risco sexual (consumo de álcool e drogas e parceiros ocasionais), assim como uma menor vigilância de saúde, incluindo a realização do teste de HIV. Alguns estudos confirmam que intervenções na área da educação aumentam os conhecimentos e a consciência sobre os riscos sexuais e influenciam positivamente as atitudes e os comportamentos face às medidas de prevenção, nomeadamente a aceitabilidade da citologia vaginal e a adesão à vacinação contra o HPV (Bruni et al., 2015).

A área de estudos teve uma influência clara nas pontuações de conhecimento SSR, resultado era espectável uma vez que as questões de sexualidade fazem parte do currículo de diversos programas das licenciaturas da área das ciências da vida e saúde (enfermagem, veterinária e biologia, entre outros), conforme também documentado noutros estudos (Santos et al., 2018, Castro et al, 2020).

Embora reconhecendo a importância do conhecimento dos jovens para tomar decisões sobre a vida sexual, em particular na prevenção de comportamentos de risco sexual (Kritsotakis et al., 2016), nem sempre o conhecimento determina o melhor comportamento. Este facto pode explicar a falta de associação que verificamos no presente estudo entre o nível de conhecimento e o uso consistente do preservativo.

Considerando o referencial teórico, os resultados do presente trabalho não deixam de ser preocupantes as lacunas de conhecimento sobre questões básicas são significativas, pois embora diversos estudos apontem para um desfasamento entre cognições, informação e comportamento, o que explica que pessoas com um nível adequado de conhecimento sobre sexualidade nem sempre adote comportamentos seguros (Kirby et al., 2011; Reis et al., 2013; santos et al., 2017). A título de exemplo existe um número considerável de estudantes que não sabem a diferença entre ser seropositivo e ter AIDS ou desconhecem que o sexo anal é de maior risco que o vaginal para a transmissão de ISTS.

Os resultados do presente estudo refletem a necessidades de se continuar a investir na educação sexual dos jovens no ensino superior, visando aumentar o conhecimento e reforçar as competências pessoais e psicossociais sobre sexo seguro, numa atitude de responsabilidade social e de cidadania, que se impõe por questões de saúde. 


\section{CONHECIMENTO SOBRE SAÚDE SEXUAL E REPRODUTIVA EM ESTUDANTES DO ENSINO SUPERIOR}

\section{CONCLUSÕES}

Face aos resultados foi possível verificar que os estudantes apresentam um nível mediano de conhecimento sobre SSR (23 pontos em 44 possíveis). São as raparigas, os estudantes mais jovens e que estudam em áreas das ciências da vida e da saúde que apresentam melhores níveis de conhecimento sobre SSR. 0 nível de conhecimento mais elevado está também associado a uma maior utilização dos serviços de SSR e a uma menor adesão a comportamentos de risco sexual, embora não tenha sido associado a uma utilização mais consistente do preservativo. Aumentar o nível de conhecimento dos estudantes sobre SSR parece ser importante para melhorar a adesão a comportamentos de proteção e diminuir o risco sexual e reprodutivo. Neste contexto as instituições de Ensino Superior enquanto agentes de conhecimento, divulgação de saberes, desenvolvimento de competências, investigação científica e inovação, podem contribuir também para a promover a Saúde sexual e reprodutiva, influenciando de forma positiva o bem-estar, a saúde e qualidade de vida dos seus estudantes.

\section{REFERÊNCIAS BIBLIOGRÁFICAS}

Asare, M. (2015). Using the theory of planned behavior to determine the condom use behavior among college students. American Journal of Health Studies, 30(1), 43-50. Retrieved from https://www.ncbi.nlm.nih.gov/pmc/articles/PMC4621079/

Bruni, L., Barrionuevo-Rosas, L., Albero, G., Aldea, M., Serrano, B., Valencia, S., ... Castellsagué X. (2015). ICO Information Centre on HPV and Cancer (HPV Information Centre). Human Papillomavirus and Related Diseases in the World. Summary Report 2015- 04-08.

Castro JF, Almeida CM, Rodrigues VM. A (des)educação contraceptiva dos jovens universitários. Acta Paulista de Enfermagem. 2020;33:eAPE201901306. Available from: http://dx.doi.org/10.37689/actaape/2020A001916

Forsyth, R., Purcell, C., Barry, S., Simpson, S., Hunter, R., McDaid, L., Elliot, L., Bailey, J., Wetherall, K., McCann, M., Broccatelli, C., Moore, L., \& Mitchell, K. (2018). Peer-led intervention to prevent and reduce STI transmission and improve sexual health in secondary schools (STASH): protocol for a feasibility study. Pilot and feasibility studies, 4, 180. https://doi.org/10.1186/s40814-018-0354-9

Garcia, T.A., Litt, D.M., Davis, K.C., Norris, J., Kaysen, D. \& Lewis, M.A. (2019). Growing Up, Hooking Up, and Drinking: A Review of Uncommitted Sexual Behavior and Its Association With Alcohol Use and Related Consequences Among Adolescents and Young Adults in the United States. Frontiers in Psychology, 10,1872. https://doi.org/10.3389/fpsyg.2019.01872

Geary, R. S., Gómez-Olivé, F. X., Kahn, K., Tollman, S., \& Norris, S. A. (2014). Barriers to and facilitators of the provision of a youth-friendly health services programme in rural South Africa. BMC health services research, 14, 259. https://doi.org/10.1186/1472-6963-14-259

Hair, J. F., Black, W. C., Babin, J. B., \& Anderson, R. E. (2014). Multivariate data analysis (7th ed.). Edinburgh: Pearson Educational.

Hartney, T., Westrop, S. J., Anderson, J., Brigstock-Barron, O., Hadley, A., Guthrieet, L., ... Connor, N. (2015). Health promotion for sexual and reproductive health and HIV: Strategic action plan, 2016 to 2019. London: Public Health England. Retrieved from

https://www.gov.uk/government/uploads/system/uploads/attachment_data/file/488090/SRHand HIVStrategicPlan_211215.pdf

Kann, L., McManus, T., Harris, W., Shanklin, S.L., Flint, K., Hawkins, J., et al. (2018).Youth Risk Behavior Surveillance - United States, 2017. MMWR Surveill Summ 2018, 67 (8), 1-479.

Khadr, S. N., Jones, K. G., Mann, S., Hale, D. R., Johnson, A. M., Viner, R. M., Mercer, C. H., \& Wellings, K. (2016). Investigating the relationship between substance use and sexual behaviour in young people in Britain: findings from a national probability survey. BMJ open, 6(6), e011961. https://doi.org/10.1136/bmjopen-2016-011961 
Kilwein, T. M., \& Looby, A. (2018). Predicting risky sexual behaviors among college student drinkers as a function of event-level drinking motives and alcohol use. Addictive behaviors, 76, 100-105. https://doi.org/10.1016/j.addbeh.2017.07.032

Kirby, D., Coyle, K., Alton, F., Rolleri, L., \& Robin, L., (2011). Reducing adolescent sexual risk. Theoretical guide for developing and adapting curriculum-based programs. California: ETR Associates.

Kritsotakis, G., Psarrou, M., Vassilaki, M., Androulaki, Z., \& Philalithis, A. E. (2016). Gender differences in the prevalence and clustering of multiple health risk behaviours in young adults. Journal of Advanced Nursing, 72(9), 2098-2113. doi:10.1111/jan.12981

Kuperberg, A., \& Padgett, J. E. (2015). Dating and hooking up in college: Meeting contexts, sex, and variation by gender, partner's gender, and class standing. Journal of Sex Research, 52(5), 517-531. https://www.ncbi.nlm.nih.gov/pubmed/24750129.

Ndugwa Kabwama, S., \& Berg-Beckhoff, G. (2015). The association between HIV/AIDS-related knowledge and perception of risk for infection: a systematic review. Perspectives in public health, 135(6), 299-308. https://doi.org/10.1177/1757913915595831

Oliveira, A.C., Caramelo, F., Patrício, M., Camarneiro, A.P., Cardoso, S.M., \& Pita JR. (2017). Impacto de um programa de intervenção educativa nos comportamentos sexuais de jovens universitários Revista Enfermagem Referência, IV Serie;(13):71-82.

Pama, M. \& Neves, J. (2013). Contraceção in Ginecología básica e familiar. J. Neves. Lisboa: Lidel, 59-65.

Picot, J., Shepherd, J., Kavanagh, J., Cooper, K., Harden, A., Barnett-Page, E., Jones, J., Clegg, A., Hartwell, D., \& Frampton, G. K. (2012). Behavioural interventions for the prevention of sexually transmitted infections in young people aged 13-19 years: a systematic review. Health education research,27(3), 495-512. https://doi.org/10.1093/her/cys014

Reis, M., Ramiro, L., Matos, M. G., \& Diniz, J. A. (2013). Determinants influencing male condom use among university students in Portugal. International Journal of Sexual Health, 25(2), 115-127. doi:10.1080/19317611.2012.728554.

Sales, W.B., Caveião, C., Visentin, A., Mocelin, D., Costa, P.M. \& Simm. E.B. (2016). Comportamento sexual de risco e conhecimento sobre IST/SIDA em universitários da saúde. Revista Enfermagem Referência IV Serie (10):19-2.

Santos, M.J., Ferreira, E., Duarte, J., \& Ferreira, M. (2017). Risk factors that influence sexual and reproductive health in Portuguese university students. Internacional Nursing Review, 65, 225-233. Doi: 10.1111/inr.12387.

Santos, M.J.0., Ferreira, E.M.S. \& Ferreira, M.M.C. (2018). Contraceptive behavior of Portuguese higher education students. Revista Brasileira de Enfermagem, 71(Suppl 4):1706-13. http://dx.doi.org/10.1590/0034-7167-2017-0623

Shepherd, J., Kavanagh, J., Picot, J., Cooper, K., Harden, A., Barnett-Page, E., ... Price, A. (2010). The effectiveness and cost-effectiveness of behavioural interventions for the prevention of sexually transmitted infections in young people aged 13-19: a systematic review and economic evaluation. Health Technology Assessment, 14(7), 1-206. http://dx.doi.org/10.3310/hta14070

Sohbet, R., \& Geçici, F. (2014). Examining the Level of knowledge on sexuality and reproductive health of students of gaziantep university. Sexuality \& Disability, 32(1), 75-84. http://dx.doi.org/10.1007/s11195-013-9332-6

Tyler, C. P., Whiteman, M. K, Kraft, J., Zapata, L., Hillis, S., Curtis, K., .. Marchbanks, P.A. (2014). Dual use of condoms with other contraceptive methods among adolescents and young women in the United States. Journal of Adolescent Health, 54(2), 169-175. http://dx.doi.org/10.1016/j.jadohealth.2013.07.042

Valentim, 0.M., Moutinho, L.S. \& Carvalho, J.C. (2021). Consumo de bebidas alcoólicas e binge drinking nos jovens em formação. Acta Paulista Enfermagem, 34: eAPE01991.

http://dx.doi.org/10.37689/actaape/2021A001991 
Voisin, D., King, K., Schneider, J., Diclemente, R., \& Tan, K. (2012). Sexual sensation seeking, drug use and risky sex among detained youth. Journal of AIDS \& Clinical Research, S1, 1-5. http://dx.doi.org/10.4172/21556113.S1-017

Wesche, R., Lefkowitz, E. S., \& Maggs, J. L. (2021). Short-Term Consequences of Sex: Contextual Predictors and Change Across College. Archives of sexual behavior, 50(4), 1613-1626. https://doi.org/10.1007/s10508020-01874-w 